\title{
Complete absence of external genitalia in limb-body wall complex: two cases
}

\author{
AVIVA LITWIN, PAUL MERLOB, AND MICHAEL GRUNEBAUM \\ From the Departments of Neonatology and Pediatric Radiology, Beilinson Medical Center and Sackler \\ School of Medicine, Tel-Aviv University, Tel Aviv, Israel.
}

SUMmARY Two neonates with limb-body wall complex (LBWC) and complete absence of the external genitalia are presented. Our patients are the sixth and seventh cases of complete absence of the external genitalia recorded in English publications and the first two cases associated with LBWC. The incidence of complete absence of the external genitalia in our newborn population during a period of 12 years (1975 to 1986) was one case per 13420 births.

The diagnosis of limb-body wall complex (LBWC) is based upon the presence of lateral body wall defects with evisceration of organs, ipsilateral limb reduction anomaly, and neural tube defects. ${ }^{1}$ These structural defects are thought to be due to early amnion rupture and the different combination of anomalies described depends on the time at which the event took place. ${ }^{23}$ We describe two patients with LBWC who present some peculiar anomalies not described in previous reports of this complicated fetal malformation.

\section{Case reports}

CASE 1

Case 1, who was born in July 1986 at 27 weeks' gestation, was the fourth infant of unrelated Ashkenazi Jewish parents, a 33 year old mother and 37 year old healthy father. There were no known malformations in the family. Two years before the present pregnancy, the mother underwent a laparotomy because of a retroperitoneal tumour which ended with resection of the tumour, extended right hemicolectomy, and end to side ileotransversostomy. Pathological examination was compatible with the diagnosis of a benign mesenchymal tumour. Because of chronic secondary diarrhoea which started shortly after the operation, the mother was treated continuously with cholestyramin ( $4 \mathrm{~g}$ twice a day) during the first and second trimester of pregnancy and occasionally during the second trimester with tinctura opii (20 gtt per day). From time to time during the second trimester she also

Received for publication 3 March 1987.

Revised version accepted for publication 9 June 1987. used papaverine (one tablet per day), a mixture of aluminium hydroxide gel (10 cc per day), and dipyrone (1 tablet per day), because of abdominal pain. There was no history of any other medication, alcohol intake, smoking, fever, or viral disease during pregnancy. During the first trimester of pregnancy she had a few episodes of vaginal bleeding treated by bed rest. The mother gained no weight and her abdomen was small. Fetal movements were first noted in the 17th week of gestation and were reduced compared to previous pregnancies. The first ultrasonographic examination performed in the 16th week of gestation revealed a single fetus and repeated sonographic examinations showed oligohydramnios and severe intrauterine growth retardation. At the age of 24 weeks of gestation ultrasound examination confirmed the oligohydramnios and intrauterine growth retardation and revealed the presence of multiple congenital anomalies including intracranial ventricular dilatation, the presence of the heart outside the thoracic cavity, and renal and bladder agenesis. Fetal echocardiography showed a normal heart configuration.

Because of the severe malformations induction of labour with prostaglandins was performed at 27 weeks' gestation. An infant of undetermined sex with eviscerated internal organs connected with the placenta and membranes was born (fig 1 ). The baby died shortly after birth. Physical examination revealed a very small head circumference $(20.8 \mathrm{~cm}$, -3 SD below the mean for gestational age ${ }^{4}$ ) and large fontanelles but normal sutures. The face showed a high forehead, low set ears, depressed nasal tip, and a deep philtrum (fig 2). The neck was normally shaped with an extraposterior cervical skin fold. The chest circumference was $12.8 \mathrm{~cm}$ 


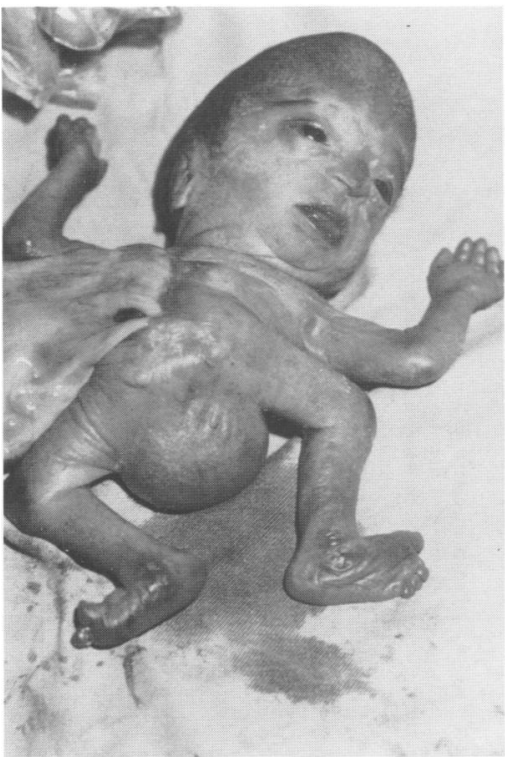

(a)

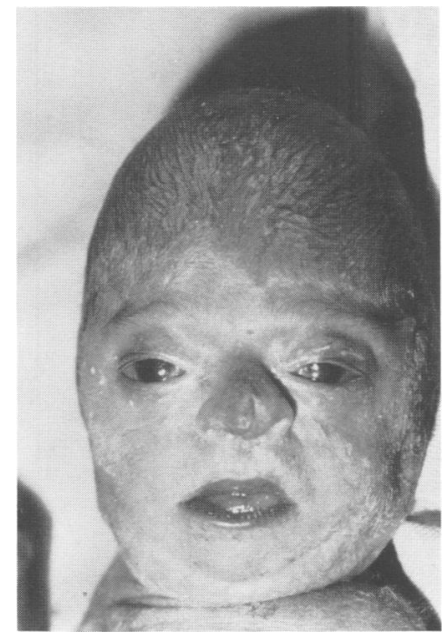

FIG 2 Face of patient 1 showing high forehead, low set ears, depressed nasal tip, and deep philtrum.

(considerably below -2 SD from the mean for gestational age $=18.5 \mathrm{~cm}^{4}$ ) with severe thoracic kyphoscoliosis.

The internal abdominal organs including the stomach, liver, spleen, kidneys, small intestine, and large bowel were eviscerated through a large defect in the right abdominal wall. A pulsating heart was outside the thoracic cavity, located in the epigastric region. The abdominal wall defect was continuous with the amnion and placenta and the internal
FIG 1 Patient 1. (a) Anterior view showing lateral body wall defect with continuity of amniotic membranes, complete absence of external genitalia, and a large sacroperineal mass. (b) Posterior view. Note the malposition with $180^{\circ}$ rotational deformity of lower limbs.

organs were eviscerated into the extracoelomic cavity.

A short umbilical cord was on the left of this mass. Upper limb anomalies included ankylosis of the elbows and clinodactyly of the right index finger. There was malposition with $180^{\circ}$ rotational deformity of the lower limbs and ankylosis of both knees, bilateral calcaneovalgus, rockerbottom feet, and right hammer toes. The anogenital area showed complete absence of genitalia and anus. Two bony prominences were substituted for the absent coccyx and a large mass of $5 \mathrm{~cm}$ in diameter was present in the sacroperineal region. Palpation of this mass revealed fluid alternating with more solid areas.

Transillumination of the skull was normal. Transillumination of the sacroperineal mass confirmed the presence of fluid. There was no oesophageal anomaly as shown by easy insertion of a nasogastric feeding tube. Chromosomal analysis showed a normal male $(46, X Y)$ karyotype. Permission for necropsy was refused.

\section{Radiographical examination}

The postmortem radiograph of the infant showed a huge soft tissue mass coming off the right abdominal wall and extending from below the right thoracic cage down to the pubis (fig 3 ). The bony skeleton itself showed malformations and deformations. The skull was turricephalic. The torso was short and narrow and no organs could be identified in the chest or abdominal cavities. The long bones of the upper and lower extremities were normal. The most 


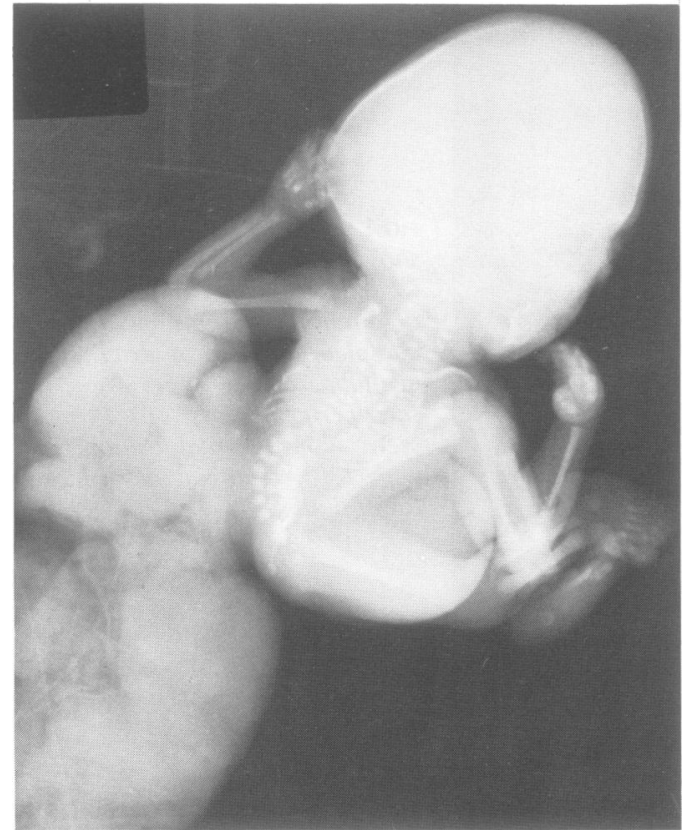

FIG 3 Radiograph of patient 1 showing malsegmentation of thoracolumbar vertebrae, absence of sacrum, maldevelopment of pelvic bones, and normal long bones of the extremities.

severe malformations were seen along the thoracolumbar spine with malsegmentation of the vertebrae, absence of the sacrum, and maldevelopment of the pelvic bones. Modulation abnormalities were also seen along the ribs on both sides.

\section{CASE 2}

Case 2 was born in November 1984 at 25 weeks' gestation to a 21 year old G2, P1, A1 healthy mother. The parents were unrelated. There was no history of any medication, alcohol intake, smoking, or fever during pregnancy. At 23 weeks' gestation the mother was admitted to hospital because of massive vaginal bleeding. Ultrasonographic examination done at the same time revealed a single fetus, oligohydramnios, hydrocephalus, and agenesis of the kidneys. As a result, induction of labour with prostaglandins was performed at 25 weeks' gestation. An infant weighing $570 \mathrm{~g}$ of undetermined sex was born and died shortly after birth. Physical examination revealed a normal head circumference $(22.4 \mathrm{~cm})$, large fontanelles with widely open sutures, a large omphalocele, anal atresia, a rudimentary left leg with hallux and nail, and complete absence of the external genitalia. Chromosomal analysis showed a normal female

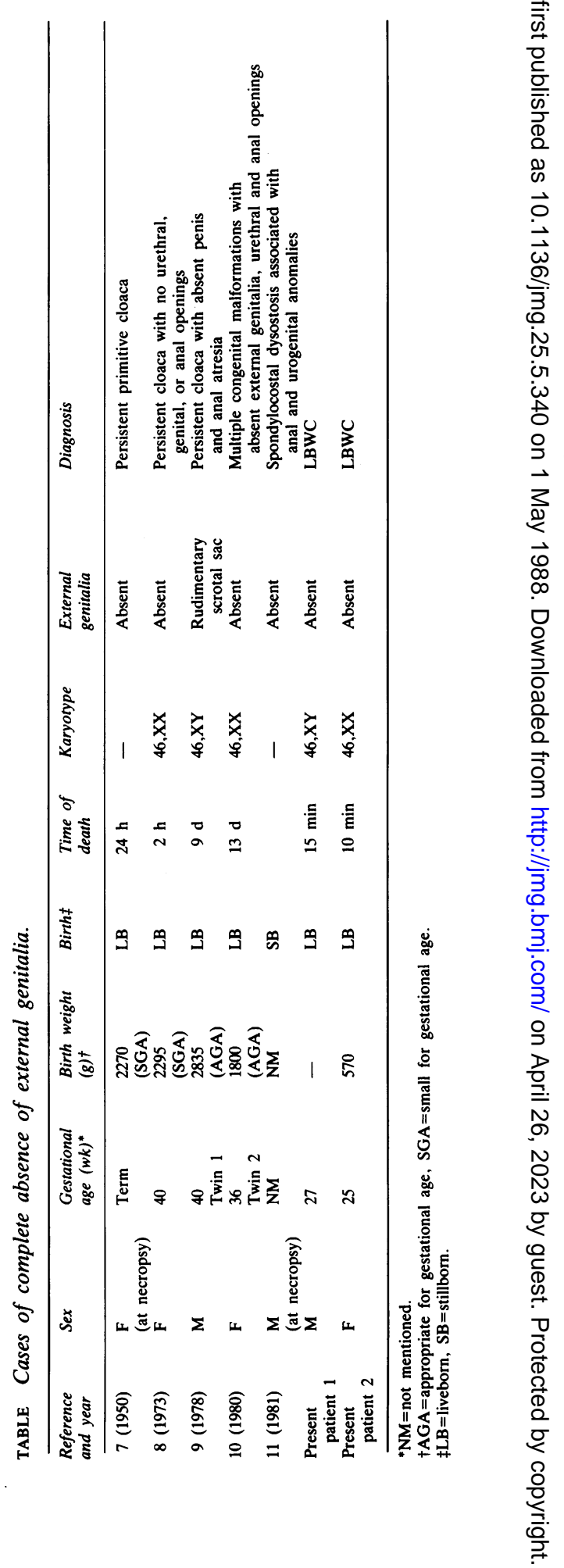


$(46, \mathrm{XX})$ karyotype. Permission for necropsy was refused.

\section{Discussion}

Limb-body wall complex (LBWC) is a term currently used to describe the combination of lateral body wall defects, limb reduction abnormalities, and neural tube defects. ${ }^{1}$ The diagnosis is based upon the presence of at least two of these three features. ${ }^{5}$

Our first patient had limb anomalies and a lateral abdominal wall defect with evisceration of the internal organs, essential for the diagnosis, without recognisable neural tube defects (compatible with group 3 of the Pagon classification ${ }^{6}$ ). In spite of the normal radiological configuration of the long bones of the upper and lower extremities, the rest of the bony skeleton showed severe abnormalities: malsegmentation of the vertebrae, absence of the sacrum, and maldevelopment of the pelvic bones.

Our second patient had a midline abdominal wall defect, limb reduction anomaly, and hydrocephalus (compatible with group 2 of the Pagon classification ${ }^{6}$ ).

Clinical findings in the present infants not previously described in LBWC include complete absence of the external genitalia in both neonates and sacroperineal mass (possibly dermoid cyst or teratoma), severe microcephaly, and extraposterior cervical skin fold in the first newborn.

Complete absence of the external genitalia is believed to be a very rare congenital malformation. Only five cases of multiple congenital malformations with complete absence of the external genitalia (both sexes) have been recorded in English publications (table). Our patients are the sixth and seventh cases of complete absence of the external genitalia and the first two associated with LBWC. During a period of 12 years (1975 to 1986) we observed three cases (including the two described) of complete absence of the external genitalia in our newborn population giving an incidence of one case per 13420 births. Thus, it appears that this malformation probably has a low level of ascertainment in current publications. Complete absence of external genitalia is not an isolated malformation; it is always part of a multimalformed infant. It has been described as part of the Potter sequence, ${ }^{9}$ sirenomelia, ${ }^{2}$ persistent primitive cloaca, ${ }^{8-10}$ spondylocostal dysplasia, ${ }^{11}$ and VATER association. ${ }^{12}$ It is important to emphasise that not all reported cases referred exactly to complete absence of the external genitalia, as in the patient of Koffler et a ${ }^{9}$ who had a rudimentary scrotal sac.

The cause of LBWC is still unknown. The possibility of a teratogen acting early in embryogenesis has not been confirmed. Although there was a history of medication during the pregnancy of our first patient, the drugs used by the mother are not known as teratogens in humans. Cholestyramin is not absorbed from the intestine and does not pass through the placenta. ${ }^{13}$ The mixture of aluminium hydroxide gel, papaverine, dipyrone, and tinct opii were used only during the second trimester of pregnancy and in negligible amounts. Today, most authorities believe that LBWC results from early amnion rupture between the third and fifth weeks of embryogenesis ${ }^{1-3}$ with transient loss of amniotic fluid. ${ }^{1}$ A number of animal experimental studies showed that complete removal of amniotic fluid early in embryogenesis produces a combination of abnormalities similar to those described as part of LBWC. ${ }^{1}$ The defects are produced either by vascular disruption ${ }^{3}$ or mechnical compression. ${ }^{1}$ The basic faulty morphogenesis of LBWC appears very early, at a stage before the two genital swellings have fused to form the midline genital tubercle. This may be an explanation for the association of complete absence of the external genitalia in our two patients with LBWC.

\section{References}

${ }^{1}$ Miller ME. Structural defects as a consequence of early intrauterine constraint: limb deficiency, polydactyly, and body wall defects. Semin Perinatol 1983;7:274-7.

2 Smith DW. Recognizable patterns of human malformation. 3rd ed. Philadelphia: Saunders, 1982:486-96.

3 Van Allen MI. Fetal vascular disruptions: mechanisms and some resulting birth defects. Pediatr Ann 1981;10:219-33.

4 Merlob P, Sivan Y, Reisner SH. Anthropometric measurements of the newborn infant (27 to 41 gestational weeks). White Plains, New York: March of Dimes, 1984.

5 Patten RM, Van Allen M, Mack LA, et al. Limb-body wall complex: in utero sonographic diagnosis of a complicated fetal malformation. AJR 1986;146:1019-24.

6 Pagon RA, Stephens TD, McGillivray BC, et al. Body wall defect with limb reduction anomalies: a report of fifteen cases. Birth Defects 1979;15(5A):171-85.

7 Kirschbaum JD. Congenital absence of external genitals (persistent primitive cloaca). J Pediatr 1950;37:102-5.

${ }^{8}$ Fitch N, Artinian B. Persistent cloaca with no urethral, genital or anal openings. Teratology 1973;8:267-72.

${ }^{9}$ Koffler H, Aase JM, Papile LA, Coen RW. Persistent cloaca with absent penis and anal atresia in one of identical twins. $J$ Pediatr 1978;93:821-3.

10 Sinniah D, Manuel JK. A syndrome of multiple congenital malformations with absent external genitalia, urethral and anal openings in one of twins. Singapore Med J 1980;21:534-6.

11 Casamassima AC, Morton CC, Nance WE, et al. Spondylocostal dysostosis associated with anal and urogenital anomalies in a Mennonite sibship. Am J Med Genet 1981;8:117-27.

12 Weaver DD, Mapstone CL, Yu PL. The VATER association. Am J Dis Child 1986;140:225-9.

13 Goodman LS, Gilman A. The pharmacological basis of therapeutics. 6th ed. London: MacMillan, 1980:842.

Correspondence and requests for reprints to $\mathrm{Dr}$ Aviva Litwin, Department of Neonatology, Beilinson Medical Center, Petah Tikva 49 100, Israel. 